\title{
Carnets
}

Revue électronique d'études françaises de l'APEF

Deuxième série - 5 | 2015

Imaginaires de guerre et autres conflits

\section{Représentations des rébellions des patriotes dans les littératures québécoise et française du XIX ${ }^{\mathrm{e}}$ siècle}

Louis Fréchette et Jules Verne

\section{Stéphanie Chifflet}

\section{(2) OpenEdition}

Journals

Édition électronique

URL : http://journals.openedition.org/carnets/381

DOI : $10.4000 /$ carnets.381

ISSN : 1646-7698

Éditeur

APEF

Référence électronique

Stéphanie Chifflet, « Représentations des rébellions des patriotes dans les littératures québécoise et française du XIX siècle », Carnets [En ligne], Deuxième série - 5 | 2015, mis en ligne le 30 novembre 2015, consulté le 19 avril 2019. URL : http://journals.openedition.org/carnets/381 ; DOI : 10.4000/ carnets.381

Ce document a été généré automatiquement le 19 avril 2019.

\section{(c) (9) \&}

Carnets est mis à disposition selon les termes de la licence Creative Commons - Atribution - Pas d'utilisation commerciale 4.0 International. 


\section{Représentations des rébellions des patriotes dans les littératures québécoise et française du XIX ${ }^{\mathrm{e}}$ siècle}

Louis Fréchette et Jules Verne

Stéphanie Chifflet

\section{Introduction}

1 L'histoire du Québec, territoire de l'ancienne « Nouvelle-France », n'est pas toujours très bien connue des Français et ce depuis la conquête anglaise, la France ayant alors délaissé ces « quelques arpents de neige $»^{1}$. Pourtant, l'un des auteurs les plus prolifiques du XIX siècle français, Jules Verne, a publié en 1888 un roman consacré à des événements, alors récents, majeurs dans l'histoire du Canada et de ce qui allait être appelé par la suite le Québec: les Rébellions des Patriotes. En effet, durant les années 1837 et 1838, un mouvement réformiste et indépendantiste s'est développé ${ }^{2}$ - notamment en politique - et a conquis une partie de la population franco-canadienne, bien décidée ainsi à se libérer de la colonisation et de la répression anglaises. Des combats armés ont ainsi eu lieu entre les Patriotes (parfois également appelés «les Fils de la Liberté ») et les troupes anglaises tentant de décourager les Rébellions, exécutant plusieurs conspirateurs importants tels De Lorimier, devenu dès lors une figure héroïque de l'histoire québécoise. Jules Verne, s'inspirant ainsi de faits historiques, conçoit les aventures d'une famille (la «FamilleSans-Nom », c'est-à-dire la famille Morgaz) entraînée dans ces soubresauts de l'histoire.

Bien évidemment, les Rébellions des Patriotes ont inspiré nombre d'auteurs francocanadiens, puis québécois, comme Louis Fréchette par exemple qui, en 1888, publie un recueil de poèmes intitulé La Légende d'un peuple et dont la troisième partie est consacrée aux Patriotes ${ }^{3}$. 
3 Nous verrons dans le présent article comment la scission effective entre deux « camps » (Patriotes, réformistes et indépendantistes, d'un côté, et les Anglais et leurs alliés, de l'autre), investit l'imaginaire des Rébellions des Patriotes. Ce dernier est un imaginaire foncièrement ambivalent, animé par un jeu constant de forces contraires. La confrontation belliqueuse fracture le Canada d'alors tant aux niveaux politique, géographique et social qu'au niveau symbolique. Les représentations des Rébellions des Patriotes sont empreintes de symboliques diverses et la littérature nous permet justement d'en identifier les éléments moteurs.

\section{Un imaginaire de la contamination}

\section{De la souillure}

4 Le roman Famille-Sans-Nom de Jules Verne est habité par la souillure. La «Famille-SansNom », dont sont issus les personnages principaux, est entachée par la faute initiale du père. En effet, celui-ci, Simon Morgaz, avocat ayant participé à la préparation d'un premier soulèvement patriotique en 1825, a, après la sollicitation de l'agent Rip (agent d'enquêtes privé anglo-canadien), trahi ses compagnons de conspiration. Alors en manque d'argent car la «passion [du jeu] le dévorait ${ }^{4}$ » (Verne, $\left.2014: 29\right)$, Simon Morgaz est déjà en partie rongé par le vice du jeu et il achève sa contamination en commettant la faute ultime de trahison ${ }^{5}$. Le jour du procès des conspirateurs, la trahison de Simon Morgaz est révélée et provoque un "mouvement de répulsion» (Verne, 2014 : 33). La réaction de ses camarades est immédiate: "Nous demandons que Simon Morgaz soit chassé de ce banc, honoré par notre présence, déshonoré par la sienne !... Nous ne voulons pas être souillés plus longtemps du contact de cet homme !» (Verne, $2014: 33$ ). À cette scène au tribunal répond une autre scène de jugement - populaire - à la fin du roman, quand les identités de Jean-Sans-Nom et de sa mère sont dévoilées : «Jamais nous ne souffrirons que la femme et le fils de Simon Morgaz souillent de leur présence le camp des patriotes!» (Verne, 2014 : 338). La famille Morgaz est ainsi marquée par la faute du père. Leur nom, connu dans tout le pays, est devenu maudit, tel le nom d'un microbe : « l'opprobre, infligé désormais au nom de Morgaz » (Verne, $2014: 35)$; « Le nom de Simon Morgaz était devenu la plus infamante des qualifications » (Verne, 2014: 36). L'infamie ${ }^{6}$ est contagieuse : «Le misérable [Simon Morgaz] était repoussé de partout. Partout on le reconnaissait, comme s'il eût porté au front quelque signe infamant, qui le désignait à la vindicte universelle » (Verne, 2014 : 37). Dans sa fuite, Simon Morgaz s'écarte du monde, obligé de se cacher pour ne pas être reconnu et lynché :

les fils achetaient quelques provisions, tandis que le père se tenait en dehors (Verne, $2014: 37$ ).

[Bridget Morgaz] baisait ses deux fils au front, comme si elle eût voulu en effacer le stigmate indélébile. (...) Était-il donc écrit, dans la conscience humaine, que, cette tache imprimée au nom d'une famille, rien ne pourrait l'effacer? (Verne, 2014 : 140).

Ah! misérables que nous sommes, dont le contact est une souillure !... (Verne, 2014 : 141).

5 À la fin du roman, Jean-Sans-Nom, c'est-à-dire Jean Morgaz, refuse tout contact tactile. Il ne veut toucher personne, pas même ces camarades de lutte : «M. de Vaudreuil offrit la main à Jean... Jean ne la prit pas. (...) Toutes les mains se tendirent vers Jean. Cette fois encore, il refusa de les toucher de la sienne » (Verne, $2014:$ 349-350). De même, Bridget 
Morgaz évite tout épanchement vis-à-vis d'une Clary de Vaudreuil pourtant encline à des élans filiaux : «Il lui paraissait naturel de l'appeler "ma mère !" Et pourtant, lorsqu'elle voulait lui prendre les mains, il semblait que Bridget cherchait à les retirer. Quand Clary embrassait Bridget, Bridget détournait brusquement la tête » (Verne, 2014 : 258).

Leur ancienne maison brûlée est également maudite, lieu infect et impénétrable. Ainsi, quand le fils Jean (Jean-Sans-Nom/Jean Morgaz) retourne sur les lieux de son enfance, il pénètre un territoire maudit :

Plus de maison à cet endroit. Sur son emplacement, rien que des ruines. Ruines sinistres, non pas celles que le temps a faites, mais celles que laisse après lui quelque violent sinistre. Et ici, on ne pouvait s'y méprendre. Des pierres calcinées, des pans de murs noircis, des morceaux de poutres brûlées, des amas de cendres, blanches maintenant, disaient qu'à une époque déjà reculée, la maison avait été la proie des flammes. (Verne, $2014: 124$ )

«Eh? Que faites-vous là, monsieur?» lui cria un vieil homme, qui venait de s'arrêter en se rendant à l'église.

Jean n'ayant point entendu, ne répondait pas.

« Eh! reprit le vieil homme, êtes-vous sourd ? Ne restez pas là !... Si l'on vous voyait, vous risqueriez d'attraper quelque mauvais compliment ! (...) Il est défendu d'entrer en cet endroit !

- Et pourquoi ?...

- Parce que c'est un lieu maudit! (...) Ne retournez pas au milieu de ces décombres! (...) [C]e serait vous souiller rien que d'en fouler les cendres. C'est ici la maison du traître !... » (Verne, 2014 : 124-125)

[L]a tradition en avait fait un lieu si infâme que personne n'osait plus l'approcher, que pas un des gens de Chambly ne l'apercevait sans lui jeter sa malédiction! (Verne, 2014 : 126).

Le retour à la maison natale est donc très funeste. La mémoire des Morgaz est elle aussi souillée, polluée par d'« infamants souvenirs » (Verne, 2014: 235): "Où Jean venait chercher des souvenirs de famille, il n'y avait que des souvenirs de honte " (Verne, 2014 : 127). C'est également à cette occasion que Jean Morgaz assiste à une scène de fureur collective prenant les allures de rite expiatoire :

Là, devant les ruines de la maison du traitre, un grand feu venait d'être allumé. Des hommes, auxquels se joignirent bientôt des enfants et des femmes, attisaient ce feu, en y jetant des brassées de bois mort.

En même temps que les cris d'horreur, ces mots de haine retentissaient dans l'air :

«Au feu, le traître !... Au feu, Simon Morgaz !»

Et alors, une sorte de mannequin, habillé de haillons, fut traîné vers les flammes. Jean comprit. La population de Chambly procédait, en effigie, à l'exécution du misérable (...). Saisi d'horreur, Jean voulu fuir... Il ne put s'arracher du sol, où il semblait que ses pieds restaient irrésistiblement attachés. Là, il revoyait son père, accablé d'injures, accablé de coups, souillé de la boue que lui jetait cette foule, en proie à un délire de haine. Et il lui semblait que tout cet opprobre retombait sur lui, Jean Morgaz (Verne, 2014 : 132).

Le dégoût face à l'acte commis est matérialisé par la boue lancée sur le mannequin en flammes. Il est, littéralement, une « ordure ». Le feu nettoie la souillure en même temps qu'il représente le bûcher symbolique de Simon Morgaz. De même, à la fin du roman, la véritable identité de Bridget Morgaz ayant été dévoilée, les Patriotes sont prêts à la lyncher. Ils lui jettent notamment "de la boue au visage", "Ses vêtements étaient déchirés et souillés " (Verne, $2014: 329)$. Ses cheveux lui cachent la figure, comme si son identité était annihilée, comme si elle était déjà symboliquement morte. La honte la ronge. 
Un député réformiste, M. de Vaudreuil, gravement blessé, est recueilli par Jean-Sans-Nom dans la nouvelle demeure de sa mère Bridget Morgaz. M. de Vaudreuil ignore leur véritable identité, dissimulée par cette famille honnie qui se sait "contaminée " par la trahison du père : «S'il [M. de Vaudreuil] apprenait quelle maison lui avait donné asile, quelles mains l'avaient disputé à la mort, l'horreur ne l'emporterait-elle pas, et, dût-il se traîner sur ses genoux, ne se hâterait-il pas de fuir le contact infamant de cette famille? " (Verne, $2014: 238)^{7}$. Les Morgaz sont hantés par la contamination. La famille Morgaz, la « Famille-Sans-Nom », est ainsi une « famille flétrie » (Verne, $2014: 41$ ), pourrie.

Les traîtres sont des êtres souillés, assimilés aux ennemis, ici les Anglais et les représentants de l'ordre, également considérés à travers le prisme de la contamination. En effet, chez Jules Verne, les Anglais saouls sont « souillés de sang» (Verne, 2014 : 247). Ici, ce n'est pas le sang qui les salit (ce qui voudrait dire que le sang est impur) mais le fait d'avoir fait verser un sang pur, le sang des Patriotes. Le territoire au complet est souillé par la présence des agents de police («[le] territoire [est] infesté d'agents de la police» (Verne, 2014:288).

11 L'impureté attachée à la famille Morgaz, telle une tare héréditaire, souille en retour ceux qui n'ont pas voulu pardonner, ceux qui ne distinguent pas la faute du père des hauts faits des fils et de leur mère. $M$. de Vaudreuil et sa fille Clary ont ainsi l'impression que le camp des patriotes a été entaché par le lynchage de Bridget Morgaz et l'exclusion de Jean-SansNom : «Il leur semblait, à tous deux, qu'ils avaient été, pour ainsi dire, souillés par cette boue d'outrages jetées à la face de Bridget et de son fils » (Verne, $2014: 343$ ). Eux qui ont été cachés, soignés et protégés par les Morgaz partagent cette souillure ${ }^{8}$.

Quoique moins présent, ce thème de la souillure occupe aussi les poèmes de Louis Fréchette consacrés aux Patriotes. Dans son poème Du Calvet, l'impureté et le vice caractérisent les ennemis et les traîtres :

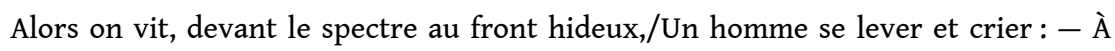
nous deux!//C'était toi, Du Calvet, qui, méprisant la rage/Du despote, osait seul tenir tête à l'orage,/Et brandir, au-dessus de tous ces fronts étroits,/À ton bras indigné la charte de nos droits.//(...)Un tyran que l'histoire a marqué du fer chaud/ Lui confisque ses biens et le jette au cachot (Fréchette, $1908: 231-232$ ).

13 La masse des Anglais ( «le spectre ») porte le stigmate de sa nature diabolique. Au baiser de Bridget Morgaz sur le front de ses fils chez Jules Verne répond ce «front hideux ». L'ennemi, «le tyran", "le despote", se distingue par cette "marque de la Bête» biblique. Cependant, chez Louis Fréchette, la souillure se manifeste à travers d'autres images. Ses poèmes sont en effet davantage imprégnés d'un imaginaire du grouillement.

\section{Du grouillement}

14 L'opposition entre la pureté et la souillure, qui traverse les représentations des Rébellions des Patriotes dans les littératures du XIX ${ }^{e}$ siècle, est en relation avec des images du grouillement. Selon Gilbert Durand, l'homme a «une répugnance primitive devant l'agitation » et « le serpent, lorsqu'il n'est considéré que comme mouvement serpentant, c'est-à-dire comme fugace dynamisme, implique lui aussi une "discursivité" répugnante qui rejoint celle des petits mammifères rapides, souris et rats" (Durand, 1969: 77). Le serpent et autres reptiles rampants sont ainsi symboliquement associés aux insectes et arachnides qui constituent ce monde grouillant, chaotique et possiblement dangereux ${ }^{9}$. Ce sont des êtres associés à la pourriture et l'on rejoint ainsi l'idée de souillure et même 
de contamination. En effet, le folklore français (dont certains pans ont émigré en Amérique du Nord) apporte maints exemples où le venin d'un serpent peut être transmis sans morsure (Sébillot, 2002 : 939-940). Le serpent, la couleuvre, la vipère portent des valeurs diverses. Les reptiles - et leurs déclinaisons merveilleuses tel le dragon - peuvent entre autres être des figures initiatrices (par exemple, saint Michel tuant le dragon) et peuvent posséder une dimension cosmique (Durand, 1969 : 363-369), mais ils peuvent être aussi des monstres dévorants. L'Église chrétienne, surtout, a particulièrement insisté sur le caractère diabolique du serpent et a grandement participé à la dévalorisation, en Occident, du serpent et des reptiles en général : ils incarnent le vice, la tentation. Comme l'illustre la poésie de Fréchette, le serpent est devenu, au XIX ${ }^{e}$ siècle, un animal essentiellement répugnant et fourbe. En effet, le poète parle du « code des vipères » (Du Calvet ; Fréchette, $1908: 231$ ) et les troupes anglaises (« les uniformes rouges») semblent ramper et se contorsionner tel un infâme serpent : «(...) de loin les uniformes rouges/ Semblaient, mouvants replis, au front des coteaux blancs,/Comme un serpent énorme aux longs anneaux sanglants " (Chénier; Fréchette, 1908: 260). La représentation reptilienne des Anglais est manifeste dans le poème Le Vieux Patriote :

Et puis n'avions-nous pas les souples, les rampants,/Les délateurs payés, les mouchards, les serpents?/Ces Judas d'autrefois, je les retrouve encore./Tout ce qui les anime et ce qui les dévore,/C'est le bas intérêt, l'instinct matériel./Ils pullulaient autour du gibet de Riel $; / /(.$.$) Il en est qui rendraient des points à la couleuvre/Pour$ faire en serpentant leur tortueux chemin (Fréchette, 1908 : 279-280).

Le symbolisme thériomorphe discrédite l'adversaire.

\section{Un décor mythique}

\section{Des lieux matriciels}

Ces êtres reptiliens grouillent et envahissent les territoires des Patriotes composés de coins et de creux. Les Patriotes sont, pour les Anglais, des ennemis invisibles, embusqués dans tous les recoins du village. Saint-Denis représente alors un cocon protecteur violemment pénétré par le régiment anglais :

Par moments, on pouvait, à travers la fumée,/Voir tout un régiment, et presque un corps d'armée,/Dans un cercle de feu, s'avancer pas à pas,/Cherchant des ennemis qu'on n'apercevait pas.//(...) Ils avaient cru n'avoir qu'à cerner un village/Avant d'y promener la torche et le pillage ;/Et voilà que battus, décimés, écharpés,/Ce sont eux qui se voient partout enveloppés!//Et comment repousser ces attaques étranges ?/Au coin des murs, au seuil des maisons et des granges,/Dans le creux des fossés, aux pentes d'un guérêt,/Où son costume gris s'efface et disparaît,/(...) Dans ce dédale affreux comment s'orienter ?... (Fréchette, 1908 : 255-256)

Chez Jules Verne, il y a un contraste constant entre l'ombre et la lumière. En particulier, la Famille-Sans-Nom relève du régime nocturne durandien, étant toujours associée aux lieux dérobés, à la nuit, au repli. Jean Morgaz, le héros Jean-Sans-Nom des Patriotes, est toujours «blotti » dans quelque recoin obscur, tapi dans l'ombre: à la taverne, il est «blotti dans le coin de la salle » (Verne, $2014: 86)$; après la défaite à Saint-Charles, il « se réfugie », avec M. de Vaudreuil inconscient, « sous le porche de l'église » puis se «blotti[t] au fond de la petite église » (Verne, 2014: 231); en fuite (sa tête est mise à prix), il ne voyage "que de nuit» et "(p)endant le jour, il se [cache] au fond des masures abandonnées, sous des fourrés presque impénétrables » (Verne, 2014 : 289); au chevet de 
M. de Vaudreuil, il «se [tient] au pied du lit, dans l'ombre, comme s'il eût cherché à ne point être vu " (Verne, 2014: 250). Enfin, il mourra lors d'une "nuit très obscure " (Verne, 2014 : 357). Sa mère, Bridget Morgaz est, à l'instar de son fils Jean, étroitement associée à la pénombre et au refuge. En public, elle apparaît :

toujours seule, toujours habillée de noir, toujours recouverte d'un long voile de veuve. Elle ne quittait que rarement sa maison (...). Quand il s'agissait d'un achat, elle attendait que la nuit ou tout au moins le soir fût venu, se glissait à travers les rues sombres, longeait les maisons, entrait rapidement dans une boutique, parlait d'une voix sourde, en peu de mots, payait sans marchander, revenait, la tête basse, les yeux à terre, comme une pauvre créature qui aurait eu honte de se laisser voir. Allait-elle à l'église, c'était dès l'aube, à la première messe. Elle se tenait à l'écart, dans un coin obscur, agenouillée, pour ainsi dire rentrée en elle-même. (Verne, $2014: 134)$

18 Quand elle sera, anonyme, dans le camp des Patriotes sur l'île Navy, Bridget Morgaz continuera de ne se sortir qu' «à la nuit tombante » (Verne, $2014: 324)$. Elle est bel et bien un être de l'ombre. La maison qu'elle occupe, «isolée » (Verne, 2014: 133), est à son image: "Modeste et triste habitation (...). Le plus souvent, la porte est fermée, les fenêtres ne sont jamais ouvertes, même derrière les volets à panneaux pleins, qui sont repoussés contre elles» (Verne, 2014: 133). Elle est d'ailleurs significativement surnommée la « Maison-Close ». Plus qu'un cloître, cette maison - la maison maternelle est un refuge, un lieu matriciel ${ }^{10}$. Bridget Morgaz mourra également dans un lieu reproduisant le cocon primordial. En effet, après leur expulsion du groupe des Patriotes (suite à la révélation de leur identité), Jean emmène sa mère « au fond d'une hutte isolée, en dehors du village de Schlosser» (Verne, 2014: 340). Ils sont retirés du monde: «Personne ne savait que cette hutte renfermait la femme et le fils de Simon Morgaz » ( Ibidem. $)^{11}$. Jean attendra que la nuit soit tombée pour enterrer sa mère dans sa terre natale aux États-Unis :

Lorsque la nuit fut venue, une nuit sombre, à peine éclairée par le «blinck » des neiges (...) Jean quitta la cabane où gisait le corps de Bridget. Puis à quelques centaines de pas, sous le couvert des arbres chargés de givre, il alla creuser une tombe avec son large couteau canadien. Sur la lisière de ce bois, perdu dans l'obscurité, personne ne pouvait le voir, et il ne voulait pas être vu (...). La neige, qui commençait à tomber, eut bientôt caché l'endroit où dormait celle qui n'était plus (Verne, $2014:$ 344).

19 La neige renforce l'image enveloppante de ce retour ultime à la terre natale. La mort de Bridget Morgaz fait écho à la mort de Simon Morgaz relatée au début du roman. Fuyant le monde avec sa famille, poursuivi par la culpabilité et la honte, il est un nouveau Caïn accablé par le regard de Dieu, « un errant parcourant la terre » (Bible, Gn 4, 14):

Ce n'était plus qu'une famille errante, à laquelle s'attachait la réprobation universelle. On eût dit que la Vengeance, une torche enflammée à la main, la poursuivait, comme dans les légendes bibliques, elle fait du meurtrier d'Abel. Simon Morgaz et les siens, ne pouvant se fixer nulle part (...). Ils couchaient, lorsqu'ils le pouvaient, au fond de cahutes abandonnées; lorsqu'ils ne le pouvaient pas, dans des anfractuosités de roches ou sous les arbres de ces interminables forêts qui couvrent le territoire.

Simon Morgaz devenait de plus en plus sombre et farouche. Il ne cessait de se disculper devant les siens, comme si quelque invisible accusateur, acharné sur ses pas, lui eût crié : traître !... traître !... (...). Mais quel lieu cherchait-il ? Sa femme ni ses fils ne pouvaient le savoir, car il allait toujours devant lui, et ils ne faisaient que le suivre.

Le 3 décembre, vers le soir, ces infortunés, exténués de fatigue et de besoin, firent 
halte dans une caverne, à demi obstruée de broussailles de ronces - quelque repaire de bête fauve, abandonnée en ce moment (...). Simon Morgaz (...) se retira au fond de la caverne (...). [B]lotti au fond, à demi couché, dans une attitude de désespoir, comme s'il eût horreur de lui-même, [il] ne faisait pas un mouvement, tandis que les reflets de la flamme éclairaient sa figure convulsée (Verne, 2014 : 36-40).

Simon Morgaz ne sortira de la caverne que pour se suicider. On distingue ici des ressemblances avec le célèbre poème La Conscience de Victor Hugo, contenu dans La Légende des siècles, qui relate l'errance paranoïaque de Caïn :

Il réveilla ses fils dormant, sa femme lasse,/Et se remit à fuir sinistre dans l'espace./ Il marcha trente jours, il marcha trente nuits./Il allait, muet, pâle et frémissant aux bruits,/Furtif, sans regarder derrière lui, sans trêve/(...). Alors il dit: - «Je veux habiter sous la terre/Comme dans son sépulcre un homme solitaire;/Rien ne me verra plus, je ne verrai plus rien. »-/On fit donc une fosse, et Caïn dit: " C'est bien!»/Puis il descendit seul sous cette voûte sombre./Quand il se fut assis sur sa chaise dans l'ombre/Et qu'on eut sur son front fermé le souterrain,/L'œil était dans la tombe et regardait Caïn. (Hugo, 1950 : 26-27)

Simon Morgaz est un personnage biblique conçu selon le modèle hugolien. Le roman de Jules Verne a ainsi des accents romantiques.

Le caractère nocturne de la Famille-Sans-Nom souligne son identité cachée, son anonymat ainsi que son sentiment de honte. Mais il y a plus. Ces "images obsédantes ", selon le terme de Charles Mauron, du coin sombre, du refuge, expriment également le lien fondamental des personnages patriotiques avec leur territoire, avec le pays à engendrer, avec la «Mère patrie »: «Une province arrachée à son pays (...), c'est un enfant arraché à sa mère ! » (Verne, $2014: 88$ ).

Enfin, la visite de l'abbé Joann à la prison où est détenu son frère a des accents de « voyage au centre de la terre ». Là encore, le décor est dépeint dans une atmosphère enveloppante, tout en clair-obscur. «[L'abbé Joann] s'assit en un coin obscur » (Verne, $2014: 295)$ puis :

[p]récédé du sergent dont le fanal éclairait ses pas, l'abbé Joann traversa la cour intérieure, au milieu de laquelle se dressait le blockhaus. Autant que le permettait l'obscurité, il cherchait à reconnaître l'étendue de cette cour (...). Puis, ils prirent par les marches d'un étroit escalier qui montait au premier étage et se développait dans l'épaisseur de la muraille. Arrivé au palier, le sergent ouvrit une porte qui se trouvait en face, et l'abbé Joann entra dans la chambre du commandant (...) médiocrement éclairée par une seule lampe (Verne, 2014 : 296-297).

L'abbé Joann se rend ensuite dans la cellule de son frère Jean :

L'abbé Joann, guidé par le sergent, redescendit l'escalier. La cellule du prisonnier occupait un angle au rez-de-chaussée du blockaus, à l'extrémité d'un couloir qui longeait la cour intérieure. Le sergent éclairant cet obscur boyau avec son fanal, arriva devant une porte basse (...). À l'intérieur, au milieu d'une profonde obscurité, couché sur une sorte de lit de camp, Jean dormait (Verne, 2014 : 300-301).

La scène figure un retour à la matrice dans laquelle ils vont répéter leur naissance, mais en inversant les identités, Jean se travestissant en abbé Joann pour pouvoir fuir la prison et participer à la révolte des Patriotes et particulièrement à l'affrontement décisif sur l'île Navy. 


\section{De l'enveloppement à l'engloutissement}

Dans Famille-Sans-Nom, l'ultime bataille menée par les Patriotes contre les Anglais se déroule sur une île, lîle Navy, près des Chutes du Niagara. Que Jules Verne choisisse de décrire spécifiquement cette bataille n'est pas anodin. Cela s'intègre parfaitement dans sa constellation d'images, relevant notamment de la symbolique matricielle (giron maternel, intimité, etc.). Selon Charles Baudouin, l'insularité est "une espèce de "Jonas" géographique » (Durand, 1969 : 273). Sur cette île Navy, les Patriotes se voient submergés et engloutis par l'ennemi anglais - avalé par le serpent. Le lieu qui était un refuge devient une prison. Enfermés, il leur est difficile d'échapper aux tirs du camp opposé. Quelquesuns vont trouver refuge sur un bateau ${ }^{12}$, sorte de prolongement de cette figure insulaire « jonasienne ». Clary de Vaudreuil et Jean Morgaz font partie de ces prisonniers marins. À l'enveloppement dans les lieux matriciels répond l'avalement, l'engloutissement. Dans cette « nuit très obscure ", Clary et Jean s'abritent sous une toile : le bateau peut encore ici être un lieu intime, un lieu de repli. Mais l'attaque des Anglais sur ce bateau, et surtout la coupure des amarres, transforment le navire en véhicule de nautonier. Le bateau, variation de la barque primordiale, n'est plus un berceau mais devient un cercueil. Jonas est remplacé par Caron. Le bateau, avec Clary et Jean à son bord, est précipité dans les Chutes du Niagara, définitivement englouti.

Que ce soit dans le creux de la terre ou dans les profondeurs marines, le roman FamilleSans-Nom de Jules Verne (comme l'ensemble de son œuvre) invite à de multiples rêveries matérielles, à des excursions au cœur de la matière. Ici, la plongée du Patriote paraît sans retour. La figure du Patriote est vouée à un destin tragique.

\section{La figure du Patriote}

\section{Fractures}

Les représentations des Rébellions des Patriotes chez Jules Verne et Louis Fréchette sont fondées sur les antagonismes et les contrastes. De multiples images figurent ainsi la fracture existant entre deux peuples : aux Franco-canadiens («les bonnets bleus») font face les Anglais («les vestes rouges» ou " uniformes rouges»); aux Catholiques, les Protestants (Verne, 2014 : 130). Dans Famille-Sans-Nom, il est d'ailleurs remarquable que l'enquêteur et espion privé anglo-canadien à la poursuite de Jean-Sans-Nom (et à l'origine de la trahison de Simon Morgaz) se prénomme Rip qui en anglais signifie « déchirer ». Son objectif est en effet de défaire (déchirer) la révolte franco-canadienne. À ces fractures nettes s'ajoutent des éléments plus ambivalents que strictement fractionnés. Les deux fils Morgaz, surtout, sont les deux faces d'un même être. Tout d'abord, ils portent le même nom, l'un en français (Jean) l'autre en anglais (Joann) ${ }^{13}$. Dès leur plus jeune âge, «Joann était d'un caractère doux, Jean d'un tempérament vif » (Verne, 2014 : 30). Dans leur même lutte patriotique, l'un a choisi les mots (Joann est devenu un prêtre au don oratoire certain) quand l'autre a choisi l'action (Jean est le meneur des Rébellions). Leur ressemblance physique est frappante au point de pouvoir les confondre: "presque les mêmes yeux, le même regard de flamme, presque la même voix et les mêmes gestes. Il [le jeune clerc Lionel] croyait revoir son héros [Jean-Sans-Nom] sous l'habit du prêtre, il croyait l'entendre... » (Verne, $2014:$ 286). C'est ainsi que Joann a pu remplacer son frère à 
la prison. Personne ne s'apercevra de la permutation et le peloton d'exécution l'abattra et l'enterrera en pensant avoir fait disparaître Jean-Sans-Nom : « Les soldats ne l'avaient pas reconnu vivant, ils ne le reconnurent pas lorsqu'il fut mort » (Verne, 2014 : 309). Joann et Jean sont un nouveau Janus. Ils sont les deux versants de la figure - mythique - du Patriote.

\section{Héroïsation et martyrologie}

Chez Jules Verne et Louis Fréchette, le Patriote est une figure héroïque. La Légende d'un peuple de Louis Fréchette est entièrement vouée à la glorification du peuple francocanadien et de ses grandes figures historiques (Du Calvet, Chénier, Papineau, etc.). Le député Papineau, en particulier, représente pour le poète le grand messager du peuple, le guide : « Il fut toute une époque, et longtemps notre race/N’eut que sa voix pour glaive et son corps pour cuirasse » (Papineau II ; Fréchette, 1908 : 252).

Chez Jules Verne, Jean-Sans-Nom, la figure exemplaire du Patriote, possède une des attributs correspondant à ses idéaux et revendications, à sa lutte nationale. En effet, simple anonyme parmi le peuple, puisque au début du roman il œuvre dans l'ombre avec l'aide de son frère Joann et de sa mère Bridget, il se distingue simplement par ses vêtements :

Que cet inconnu appartînt au parti qui luttait pour l'indépendance nationale, cela se devinait rien qu'à son costume. Vêtu à peu près comme des intrépides aventuriers auxquels on donne encore le nom de « coureurs des bois ", il portait sur la tête la "tuque" bleue, et ses vêtements - une sorte de capot, croisé sur la poitrine, une culotte d'un rude tissu grisâtre, serrée à la taille par une ceinture rouge - était uniquement en " étoffe du pays ».

Qu'on ne l'oublie pas, l'emploi de ces étoffes indigènes équivalait à une protestation politique, puisqu'il excluait les produits manufacturés, importés d'Angleterre (Verne, $2014:$ :51).

1 La façon de se vêtir est un acte militant pour le Patriote vernien. La figure du Patriote est étroitement liée à son territoire ${ }^{14}$. Ses emblèmes, surnoms et drapeau affichent sa cause indépendantiste et son identité revendiquée. En plus d'être appelés les «bonnets bleus » (face aux «vestes rouges» ou "uniformes rouges»), les Patriotes baptisent leurs compagnies de noms évocateurs tels «les Raquettes» et les «Castors », soulignant l'ancrage géographique et culturel du peuple qu'elles représentent et défendent. Enfin, les drapeaux fédèrent les Patriotes autour d'un rejet radical des colons anglais et d'une aspiration commune à l'indépendance :

Un pavillon noir, sur lequel se dessine une tête de mort avec deux os en croix, dénonce les noms de ces gouverneurs détestés, Craig, Dalhousie, Aylmer, Gosford. Enfin, à l'honneur de l'ancienne France, un pavillon blanc porte d'un côté l'aigle américain environné d'étoiles, de l'autre l'aigle canadien, tenant dans son bec une branche d'érable (Verne, $2014: 212$ ).

Le drapeau noir est, comme le signale Jean Chesnaux dans sa préface de Famille-Sans-Nom, typiquement vernien, ressemblant au drapeau du Capitaine $\mathrm{Nemo}^{15}$ ou à celui de Robur ${ }^{16}$. Il est le «symbole de la révolte contre l'ordre politique et social » (Verne, 1970 : xIV) : « Fuyez, tyrans! Le peuple se réveille!» (Verne, 2014 : 212).

En outre, chez Jules Verne, le Patriote est un héros errant. Jean-Sans-Nom est un être de l'ombre, surgissant ici et là : 
il en était un autre qui travaillait dans l'ombre et si mystérieusement que les principaux réformistes ne l'avaient jamais aperçu qu'en de rares circonstances. Autour de ce personnage s'était créée une véritable légende, qui lui donnait une influence extraordinaire sur l'esprit des masses: Jean-Sans-Nom - on ne le connaissait que sous cette appellation énigmatique (Verne, $2014: 20$ ).

Dans les masses, on le considérait comme le chef mystérieux des Fils de la liberté. Il n'apparaissait qu'à l'heure où il fallait donner de sa personne, et disparaissait ensuite pour reprendre son œuvre (Verne, $2014: 138)$. d'être enrayé dès le début. C'est pour le généraliser que j'ai visité les paroisses de l'est et de l'ouest, que je vais parcourir celles du centre. Je compte repartir cette nuit même " (Verne, 2014: 106). Alors que Jean-Sans-Nom va de maison en maison sur tout le territoire, son frère Joann va d'église en église, lui aussi d'est en ouest : « Il a parcouru les comtés de l'ouest, et partout, on s'est précipité pour l'entendre » (Verne, 2014:129). Il « [attire] à lui les populations des bourgades et des campagnes » (Verne, $2014: 138)$. Joann ne répand non plus seulement «la bonne parole » chrétienne, mais « la bonne parole » politique, indépendantiste et nationaliste. Il est un «apôtre» (Verne, 2014: 129) «poursuivant sa mission» (Verne, $2014: 106)$. Il est un soutien indispensable à la cause qu'il mène conjointement avec son frère Jean. Celui-ci est l'incarnation principale du Patriote dans le roman. Il est « le chef mystérieux », celui qui peut soulever effectivement les masses. Il est «l'âme des insurrections populaires » (Verne, 2014 : 319), le « héros de l'indépendance » indispensable pour motiver les troupes (Verne, $2014: 309$ ). Il est pétri d'une charge symbolique puissante. Lors de la bataille de l'île Navy, à la fin du roman, il compte « [tomber] comme la foudre » et faire une « réapparition miraculeuse » (Verne, 2014 : 335). Il est un "Messie» (Verne, 2014 : 117). L'héroïsation de ces personnages fictionnels prolonge l'héroïsation de personnages historiques, tel Jean-Olivier Chénier tué lors de la bataille de Saint-Eustache :

La résistance de Chénier et des siens fut héroïque. Exposés aux boulets et aux balles, ils durent se retrancher dans le presbytère, le couvent et à l'église. La plupart n'avait même pas de fusils, et, comme ils en réclamaient:

«Vous prendrez les fusils de ceux qui seront tués! » répondit froidement Chénier.

(...) Chénier se vit contraint d'abandonner l'église. Une balle le jeta à terre. Il se releva, il fit feu. Une seconde balle l'atteignit à la poitrine. Il tomba, il était mort. (... ) Dans le pays, on dit toujours : Brave comme Chénier (Verne, $2014: 321$ ).

La même scène est célébrée par Louis Fréchette :

Cent hommes tout au plus, groupe que paralyse/Le manque de fusils et de munitions./Qu'importe! dans leurs rangs nulles défections !/...) - C'est bien, leur dit Chénier un éclair aux sourcils,/Les mourants cèderont aux autres leurs fusils :/ Nous en aurons bientôt assez pour tout le monde !/(...) La scène ne dura que deux minutes, mais/Ceux qui purent la voir ne l'oublieront jamais./Le héros, en sautant du haut d'une croisée,/S'affaissa sur le sol une jambe brisée./Ce n'est rien! sous le plomb qui grêle à bout portant,/Chénier sur un genou se relève un instant ;/Il se dresse, aveuglé de sang, l'habit sordide,/Défiguré, hagard, effroyable, splendide ;/ Et, pour suprême insulte à la fatalité,/Le fier mourant cria : - Vive la liberté! ( Chénier ; Fréchette, 1908 : 261-266).

Le sacrifice auréole définitivement la destinée de Chénier d'un honneur légendaire. Il rejoint ainsi le panthéon occidental des grands révoltés de l'histoire : il est un « nouveau Spartacus » (Chénier ; Fréchette, 1908 : 260).

L'héroïsation de la figure du Patriote s'inscrit finalement dans une martyrologie. Chez Jules Verne, toute la Famille-Sans-Nom est destinée à expier la faute du père, jusqu'à la 
mort. Joann, d'abord, est «la première victime offerte en expiation du crime de Simon Morgaz » (Verne, 2104: 309). Bridget Morgaz est intraitable sur ce sujet face à son fils Jean : « Mon fils, dit Bridget, il faut souffrir !... C'est notre part ici-bas !... C'est l'expiation! ... » (Verne, $2014:$ 339). Ses dernières paroles sont explicites et confirme la portée - voire la nécessité - sacrificielle de l'engagement de Jean :

Mon fils, ton frère est mort, et moi, je vais mourir, après avoir bien souffert! Je ne me plains pas! Dieu est juste ! C'était l'expiation! Jean, pour qu'elle soit complète, il faut que tu oublies l'outrage! Il faut que tu reprennes ton œuvre! Tu n'as pas le droit de déserter !... Le devoir, mon Jean, c'est de te sacrifier pour ton pays jusqu'à ce que tu tombes (Verne, $2014: 341$ ).

En narrant, à partir de faits historiques, la vie de cette " Famille-Sans-Nom ", Jules Verne vise à souligner le caractère intrinsèquement tragique des Rébellions des Patriotes « dont les noms ne s'effaceront jamais du martyrologue de l'histoire franco-canadienne " (Verne, 2014 : 362). La figure du martyr est également présente chez Louis Fréchette, mais sans la dimension chrétienne, patente chez Jules Verne. Son œuvre poétique est la mémoire de «nos martyrs patriotes» (Hindelang; Fréchette, 1908: 275). Chez ces deux auteurs francophones du xix siècle, le héros nationaliste doit incarner la prééminence de la cause nationale et avoir pour devise : « Avant nous, il y a le pays » (Verne, 2014 : 271).

\section{Conclusion}

En définitive, les œuvres de Jules Verne et Louis Fréchette forment une grande épopée romantique («La lutte fut épique et le combat géant » [Saint-Denis ; Fréchette, 1908 : 254]; « lutte homérique» [Hindelang; Fréchette, $1908: 274]$ ]). Des accents hugoliens s'entendent chez les deux auteurs ${ }^{17}$ et Louis Fréchette, plus directement impliqué que Jules Verne dans l'histoire du futur Québec, est un véritable poète patriotique nourri par le romantisme. Ainsi, à partir de genres différents - l'un à partir d'une fiction romanesque (fondée sur des événements historiques) avec des personnages centraux inventés, l'autre à partir de poèmes glorifiant les personnages historiques ayant participé aux Rébellions Jules Verne et Louis Fréchette tissent assurément une « légende du peuple ».

Mais les deux auteurs écrivent pour des lectorats différents. ${ }^{18}$ Jules Verne, s'adressant au lectorat français, s'applique surtout à brosser un portrait de ce Canada si lointain et si méconnu. Il informe ainsi les Français du sort de cette contrée abandonnée («quelques arpents de glace »; Verne, $2014:$ 8) par la France et où pourtant des francophones luttent pour conquérir leur liberté. Cela est aussi l'occasion pour lui de mettre en scène ses idées politiques et son «attachement quarante-huitard aux droits des nationalités» (Verne, 1970 : VIII). L'intérêt est aussi pour lui, selon Jean Chesnaux, de mobiliser les Français à la cause de l'Alsace-Lorraine perdue en 1871.

41 En revanche, chez Louis Fréchette, l'enjeu est plus grand. Son œuvre participe à l'élaboration d'un grand récit fondateur et fédérateur participant à la constitution d'un imaginaire typiquement franco-canadien, puis québécois. L'imaginaire des Rébellions des Patriotes vise à consolider les fondations de la nation québécoise. Il est un événement marquant et constitue un héritage important pour l'identité nationale, chanté au fil des siècles, comme le fera l'un des plus grands chansonniers et poètes du Québec, Félix Leclerc en 1975 dans sa chanson Chant d'un patriote: "Je vais tuer sa majesté/Qui dit m'attendre qui dit m'aimer/Cent fois par jour elle me trahit/On doit mourir quand on trahit/(...) Et si demain, mains dans les fers/Vous me rejetez à l'exil/Quelqu'un viendra 
finir ma guerre/Peut-être vot' fils ainsi soit-il/Quelqu'un viendra gagner ma guerre/Peutêtre vot' fils ainsi faut-il » (Leclerc, $1996: 54-55)$.

\section{BIBLIOGRAPHIE}

BACHelard, Gaston (1957). La Poétique de l'espace. Paris : Presses Universitaires de France.

BACHELARD, Gaston (1948). La Terre et les rêveries du repos. Paris : José Corti.

BACHELARD, Gaston (1942). L'Eau et les rêves. Essai sur l'imagination de la matière. Paris : José Corti.

BIBLE (LA SAINTE) (1955). Traduction en français sous la direction de l'École biblique de Jérusalem. Paris :

Éd. du Cerf.

BIRON, Michel, DUMONT, François, NADOUT-LAFARGE, Élisabeth (2007). Histoire de la littérature

québécoise. Montréal : Boréal.

DUGAS, Marcel (1934). Un Romantique canadien : Louis Fréchette, 1839-1908. Paris : Éd. de la « Revue Mondiale ».

DURAND, Gilbert (1969). Les Structures anthropologiques de l'imaginaire. Introduction à l'archétypologie générale. Paris : Bordas.

FILTEAU, Gérard (1975). Histoire des Patriotes. Montréal : L’Aurore.

FRÉCHETTE, Louis (1908). Poésies choisies I. La Légende d'un peuple. Montréal : Librairie Beauchemin.

HUGO, Victor (1950). La Légende des siècles, vol. I. Paris : Garnier.

JAUSS, Hans Robert (1978). Pour une esthétique de la réception. Paris : Gallimard.

LECLERC, Félix (1996). Félix tout en chansons. Québec : Nuit blanche éditeur.

MAURON, Charles (1962). Des métaphores obsédantes au mythe personnel. Introduction à la

psychocritique. Paris : José Corti.

SÉBILLOT, Paul (2002). Croyances, mythes et légendes des pays de France. Paris : Omnibus.

VERNE, Jules (2014). Famille-Sans-Nom. CreateSpace Independent Publishing Platform.

VERNE, Jules (1970). Famille-Sans-Nom. Montréal : La Maison Réédition Québec Inc.

\section{NOTES}

1. Expression de Voltaire passée dans le langage courant pour désigner le désintérêt de la France vis-à-vis de son ancienne colonie nord-américaine.

2. Mais les Rébellions des Patriotes ont leurs racines au début des années 1830.

3. En 1862, Louis Fréchette avait publié une pièce de théâtre, Félix Poutré, avec un Patriote comme personnage principal.

4. Nous soulignons. 
5. D'ailleurs, sa femme et ses fils rachèteront la faute en versant l'argent ainsi gagné à la cause indépendantiste.

6. Le mot est fréquemment employé par Jules Verne.

7. À noter l'allusion récurrente aux mains.

8. Jules Verne fait ici preuve d'une morale chrétienne remarquable.

9. Voir, parmi les Plaies d'Égypte, le serpent, les grenouilles, les sauterelles dans L'Exode (Ex 7, 8 à

9, 20).

10. Sur les rêveries autour de la maison natale, voir les travaux de Gaston Bachelard.

11. Nous soulignons.

12. Fait historique.

13. Car leur mère Bridget Morgaz était originaire des États-Unis.

14. Lien représenté en partie par les lieux matriciels évoqués plus haut.

15. Vingt mille lieux sous les mers.

16. Robur le Conquérant.

17. Louis Fréchette était même surnommé "Victor Hugo le Petit ».

18. Nous nous référons ici à l'Esthétique de la réception de l'École de Constance. Voir notamment les travaux de Hans Robert Jauss.

\section{RÉSUMÉS}

Le présent article vise à définir les contours de l'imaginaire des Rébellions des Patriotes (1837-1838), conflit majeur et déterminant dans la construction identitaire de la nation québécoise, à travers deux œuvres du XIX ${ }^{\mathrm{e}}$ siècle, parues en 1888 : l'une québécoise (La Légende d'un peuple de Louis Fréchette), l'autre française (Famille-Sans-Nom de Jules Verne). De cette lecture croisée, nous avons extrait les éléments moteurs de cet imaginaire, en y décelant notamment les constantes mythiques communes aux deux auteurs. Nous nous interrogeons ainsi sur les notions de pureté et d'impureté (ce que nous avons appelé l'imaginaire de la contamination) puis sur les caractéristiques des décors verniens et fréchettiens, relevant tous deux, selon nous, d'un héritage mythique privilégiant les images matricielles. Nous terminons enfin notre étude sur la figure du Patriote, figure centrale des deux œuvres.

In this paper, we want to identify the imaginary of the Patriot's Rebellions (1837-1838), a determining and very important event for identity construction of the Québec Nation. Two perspectives will be analysed through 19th Century literature: from Quebec, Louis Fréchette's La Légende d'un peuple (1888); and from France, Jules Verne's Famille-Sans-Nom (1888). From a comparative approach, we will show the inherent mythic elements of this imaginary. We will study the notions of Purity vs Impurity (the so called imaginary of contamination) and the characteristics of Verne's and Fréchette's background, composed essentially of a mythic heritage privileging maternal images. Finally, we will discuss the Patriot character's central role in the two works. 
INDEX

Mots-clés : Québec, imaginaire, héros, mythe, épopée nationale

Keywords : Quebec, imaginary, hero, myth, national epopee

\section{AUTEUR}

\section{STÉPHANIE CHIFFLET}

Université du Québec à Chicoutimi

Stephanie_chifflet@uqac.ca 